\title{
BMJ Open Evaluation of the impact and implementation of a national clinical programme for the management of self- harm in hospital emergency departments: study protocol for a natural experiment
}

\author{
Eve Griffin (D) ,, ${ }^{1,2}$ Sheena M McHugh (D) , ${ }^{1}$ Anne Jeffers (D) , ${ }^{3}$ David Gunnell (D) ,, ${ }^{4,5}$ \\ Ella Arensman (D) , ${ }^{1,2}$ Ivan J Perry (1) , ${ }^{1}$ Grace Cully (D) , ${ }^{1,2}$ Brendan McElroy (D) , ${ }^{6}$ \\ Margaret Maxwell (D) ," Shu-Sen Chang (D) , ${ }^{7}$ Eimear Ruane-McAteer (D) , \\ Paul Corcoran (1) ${ }^{2}$
}

To cite: Griffin E, McHugh SM, Jeffers A, et al. Evaluation of the impact and implementation of a national clinical programme for the management of selfharm in hospital emergency departments: study protocol for a natural experiment. BMJ Open 2021;11:e055962. doi:10.1136/ bmjopen-2021-055962

- Prepublication history for this paper is available online. To view these files, please visit the journal online (http://dx.doi org/10.1136/bmjopen-2021055962).

Received 29 July 2021 Accepted 02 December 2021

D Check for updates

(C) Author(s) (or their employer(s)) 2021. Re-use permitted under CC BY-NC. No commercial re-use. See rights and permissions. Published by BMJ.

For numbered affiliations see end of article.

Correspondence to

Dr Eve Griffin; evegriffin@ucc.ie

\section{ABSTRACT}

Background A National Clinical Programme for the Management of Hospital-Presenting Self-Harm (NCPSH) was introduced in Ireland in 2014. This involved the development of a model of care to standardise the management of self-harm in emergency departments, to be delivered by dedicated clinical nurse specialists. The core components of the programme were to: ensure an empathic and timely response, conduct a biopsychosocial assessment, involve family members in assessment and discharge planning, and provide a bridge to next care. The overall aim of the programme was to reduce the rate of repeat self-harm. This multistage study will evaluate the impact of the NCP-SH on hospital-presenting self-harm and to identify determinants influencing its implementation.

Methods Employing a sequential mixed methods design, the first stage will use data from the National Self-Harm Registry Ireland to examine the impact of the NCP-SH on self-harm repetition, along with other aspects of care, including provision of psychosocial assessments and changes in admissions and postdischarge referrals. A cost-effectiveness analysis will assess the cost per repeat self-harm attendance avoided as a result of the NCP-SH. The second stage will identify the influences of implementation fidelity - adherence to the programme's core components-using a combination of document analysis and semistructured interviews with staff of the programme, guided by the Consolidated Framework for Implementation Research.

Ethics and dissemination This study has received full ethical approval and will run until August 2023. This study is novel in that it will identify important factors influencing successful implementation of complex programmes. It is expected that the findings will provide important learnings for the integration of mental health services in general hospital settings and will be disseminated via peer-review publications along with reports for clinicians and policymakers.
Strengths and limitations of this study

- A strength of this research is the use of a natural experiment design, utilising existing national data sources to evaluate the impact of a national clinical programme for self-harm.

- The sequential mixed methods design will use implementation science methods to identify the factors contributing to successful implementation of national programmes to improve clinical care.

- Multiple sources of data will be combined to address a potential limitation in the form of confounding, along with using an interrupted time series analytical framework.

- The study is limited by the retrospective nature of the evaluation, which will be addressed by interviewing staff who were in place during the initial stages of implementation of the programme.

\section{INTRODUCTION}

Individuals who engage in self-harm are at increased risk of suicide and other causes of premature mortality. ${ }^{12}$ While the majority of self-harm occurs in the community, particularly for adolescents, ${ }^{3}$ there are approximately 12000 presentations to hospital in Ireland each year as a result of self-harm. ${ }^{4}$ Of those, $16 \%$ will represent with further self-harm within 12 months and at least $4 \%$ will die by suicide within 5 years. ${ }^{1}$ As well as the personal costs associated with suicidal behaviour, the economic costs of hospital-presenting self-harm are substantial-estimated to be $£ 162$ million in England annually. ${ }^{5}$ As a result, these patients represent an important group in terms of preventing repeated self-harm and suicide. 
Aspects of routine care in emergency settings following self-harm, such as a comprehensive assessment of an individual's psychosocial needs and mental health status, may be associated with better outcomes such as lower rates of repeat self-harm ${ }^{67}$ and are recommended in clinical guidelines and quality standards. ${ }^{8-10}$ However, significant variation across hospitals in these routine aspects of care are well known ${ }^{11-14}$ and an audit conducted by the UK's Royal College of Emergency Medicine found that few hospitals met the target of providing patients with an assessment by mental health services within 1 hour of referral. ${ }^{15}$ This variation can be largely explained by differences in the availability of specialised mental health resources across hospitals. ${ }^{11}$ Such inconsistencies in the quality of care for self-harm can have significant negative impacts for individuals, particularly those who leave before their care has been completed, and may increase risk of repeat self-harm. ${ }^{16}$

In 2014, a National Clinical Programme for the Assessment and Management of Patients Presenting to the Emergency Department following Self-Harm (NCP-SH) was introduced in Ireland. Prior to this, only 11 of 26 hospitals in Ireland providing 24 hour care had onsite consultant-led Liaison Psychiatry services. ${ }^{17}$ The remit of these services included the provision of care for individuals presenting to emergency departments following self-harm. While guidelines for the composition of these multidisciplinary teams was provided in national policy documents, ${ }^{17} 18$ no standard operating procedures were developed. As a result, differing models of care were adopted across these services and so the provision of assessments and admission policies were not standardised. ${ }^{18}$

A model of care for the NCP-SH was developed jointly by the College of Psychiatrists of Ireland and by a working group from the Health Service Executive including clinicians, managers and people with lived experience of selfharm or suicide by a family member. ${ }^{19}$ This outlined a pathway of care for all adults, aged 18 years and over, who presented to the emergency department following self-harm or with suicidal ideation, including receiving an empathic, timely response in the emergency department, receiving a biopsychosocial assessment, ensuring family members were involved at the assessment and discharge planning and providing linkage to next appropriate care. New funding was made available for 35 clinical nurse specialists in self-harm (CNS) for all hospitals in Ireland with 24 hour emergency services and the programme was rolled out across these hospitals between 2014 and 2017. The overall aim of the programme was to achieve a reduction in the rate of repeat self-harm. ${ }^{19}$ A 2017 review of the operation of the programme reported on service availability across the participating hospitals. This review highlighted examples of good practice across all sites and outlined recommendations to ensure standardised implementation of the programme..$^{20}$ Despite the programme being in existence for more than 7 years, no formal evaluation of the impact of the programme on patient outcomes or of its implementation have been undertaken.
While initiatives such as this have the potential to increase the provision of evidence-based care, their outcomes have been under-researched. ${ }^{21}{ }^{22}$ Some recent natural experiment studies have shown how changes in service delivery impact on patterns of self-harm, including hospital reconfiguration ${ }^{23}$ and expansion of liaison psychiatry services. ${ }^{2425}$ However, there are virtually no studies examining the impact of national programmes for the clinical management of self-harm in hospital settings. ${ }^{22}{ }^{26}$ In addition, no previous study has examined how these programmes are implemented or have sought to identify determinants of successful implementation. ${ }^{27}$

The primary aim of this study will be to examine the impact of the introduction of the NCP-SH on hospitalpresenting self-harm and to identify the factors (determinants) influencing the fidelity of implementation of the programme. A mixed methods approach is adopted, whereby data from a national registry will be used to examine the impact of the NCP-SH on patient outcomes, along with a cost-effectiveness study. An interview study with staff responsible for delivering the programme will seek to identify the determinants of achieving implementation fidelity and adherence to the core components of the programme. ${ }^{28}$

\section{METHODS}

Setting

Ireland has a population of 4.8 million, according to the most recent 2016 census. ${ }^{29}$ Within the Irish healthcare system hospitals fall under seven geographical hospital groups, each with their own governance structure. The groups are constructed according to geographical locations, as well as combining hospitals varying in model status, size and specialty. There are 26 acute general hospitals, located across all seven hospital groups, that provide a 24 hour emergency department service which were eligible to implement the NCP-SH.

\section{Description of the NCP-SH}

Introduced between 2014 and 2017, the original objectives of the NCP-SH were: to improve the assessment and management of all individuals who present with self-harm to the acute hospital emergency department; to improve access to appropriate interventions at times of personal crisis; to ensure rapid and timely safe linkage to appropriate follow-up care; and to optimise the experience of families and carers in supporting those who present with self-harm. Through these objectives, the overarching aim of the NCP-SH is to reduce the overall rate of repeat selfharm among those attending hospital. ${ }^{19}$

The model of care would be delivered by 35 CNSs who would be based in emergency departments across the 26 hospitals providing 24 hour care (see above). These CNSs would receive a standardised training programme and be under the clinical supervision of a Consultant Psychiatrist, to ensure clinical and managerial governance. ${ }^{19}{ }^{30}$ These staff members would work alongside 


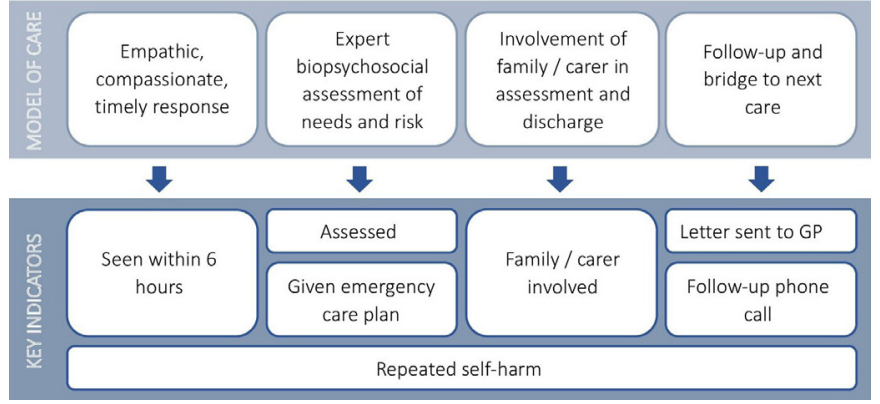

Figure 1 Core components and key indicators for the National Clinical Programme for Self-harm (NCP-SH). GP, general practitioner.

existing mental health and liaison teams, to specifically address the care and treatment required for people who present to hospital following self-harm. It would be the responsibility of the CNSs to implement the four components of the NCP-SH model of care. These are: (1) to ensure an empathic, compassionate and timely response to all patients who present to hospital following self-harm; (2) to ensure that all patients who present to hospital following self-harm receive an expert biopsychosocial assessment of needs and risks; (3) to ensure that a patient's family or carer(s) are involved in the assessment and follow-up process, where appropriate and (4) to link the patient with appropriate follow-on care, including primary care, with a view to reducing the incidence of repeat self-harm ${ }^{19}$ (see figure 1 ).

The NCP-SH identifies six key indicators which reflect the delivery of integral features of the programme. These were to be recorded, along with other data items, by programme staff on a monthly basis. These are: (1) percentage of patients receiving a biopsychosocial assessment; (2) percentage seen within 6 hours of presentation to hospital; (3) number of general practitioner letters sent within 24 hours; (4) percentage of patients receiving a follow-up phone call; (5) percentage of family/carer involvement and (6) percentage of patients receiving an emergency care $\operatorname{plan}^{19}$ (figure 1).

Between 2014 and 2017, the NCP-SH was implemented in 24 of 26 acute general hospitals in Ireland. Posts were allocated based on service demand, with a recommendation that there be one CNS available per 250 self-harm presentations annually. ${ }^{30}$ In general, hospital patients over the age of 18 years are under the remit of the NCP$\mathrm{SH}$, with children and adolescents assessed by Child and Adolescent Psychiatry teams.

The placement of the CNS in each hospital was dependent on hospital governance and existing services. Within each acute hospital, a Clinical Lead was to be identified to facilitate the delivery of the NCP-SH and provide clinical supervision to the CNS(s). In hospitals with an existing Liaison service, the CNS was to become part of that team, with the Clinical Lead being the Liaison Psychiatrist. In hospitals with no Liaison service, a named Consultant from a General Adult Psychiatry team in the region was to be identified for reporting and supervision.
National Clinical Programmes Office

Co-ordinate the implementation and monitoring of programme including management of data collection and the provision of training to CNSs

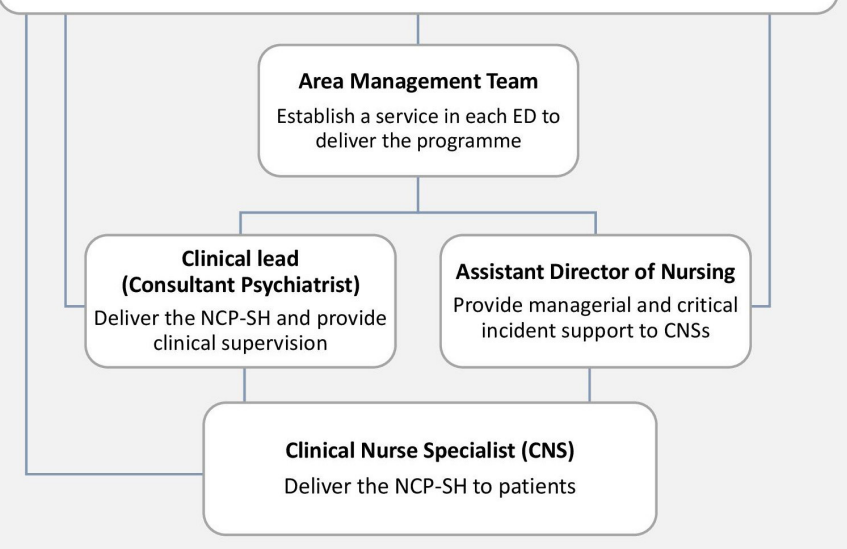

Figure 2 Governance of the National Clinical Programme for Self-harm (NCP-SH). ED, emergency department.

The CNSs were to report directly to the Area Director of Mental Health, via the Assistant Director of Nursing. Nationally, the NCP-SH is overseen by a national clinical office, consisting of the National Clinical Lead (Psychiatrist), Programme Manager, National Nurse Lead and Data Manager, who monitor the implementation of the programme via monthly data reporting, interim site evaluations and audits. This office also co-ordinates ongoing training programmes and study days for the CNSs (see figure 2$).{ }^{19}$

\section{Study design}

A mixed methods approach will be applied using a sequential explanatory design, which will comprise of two stages, where quantitative data will inform qualitative data collection and data analysis, along with subsequent integration of both quantitative and qualitative data. ${ }^{31}$ Stage 1 will be a natural experiment, ${ }^{32}$ using data from the National Self-Harm Registry Ireland (NSHRI) ${ }^{4}$ to examine the impact of the NCP-SH on patient outcomes. Measures of implementation fidelity, conceptualised here as the extent to which the core components were delivered by those responsible, ${ }^{28}$ will be included in the impact evaluation (stage 1), as a mediator between the intervention and observed outcomes. A cost-effectiveness analysis will also be undertaken. Stage 2 will be a primarily qualitative study and will begin by describing the implementation of the NCP-SH and will identify implementation strategies adopted by hospitals. As fidelity is the primary implementation outcome to be examined, the determinants (barriers and facilitators) contributing to fidelity across hospital sites will be examined, using the Consolidated Framework for Implementation Research (CFIR). ${ }^{33}$

This study will follow guidelines developed by the Medical Research Council for process evaluation of complex interventions. ${ }^{34}$ This protocol has been developed in line with the template for intervention description and replication checklist and guide..$^{35}$ 
Stage 1: evaluating the impact and cost-effectiveness of the NCP-SH

The first quantitative stage of this study will examine the impact of the implementation of the NCP-SH on hospitalpresenting self-harm. This impact will be formally tested by examining changes in repeat self-harm, along with outcomes relating to provision of care in the hospital. This stage will also consider the cost-effectiveness of the NCP-SH.

\section{Intervention outcomes}

The primary data source used to evaluate the impact of the NCP-SH will be the NSHRI, a national monitoring system of self-harm attendances to hospital emergency departments in Ireland. The standard operating procedures of the Registry have been previously described. ${ }^{36}$ The definition of self-harm used by the Registry is 'an act with non-fatal outcome in which an individual deliberately initiates a non-habitual behaviour, that without intervention from others will cause self-harm, or deliberately ingests a substance in excess of the prescribed or generally recognised therapeutic dosage, and which is aimed at realising changes that the person desires via the actual or expected physical consequences'. ${ }^{37}$ As the NCP-SH was delivered to adults, data from the Registry involving individuals aged 18 years and over will be used for the period 2004-2019, accounting for approximately 10000 of the 12000 annual self-harm presentations in Ireland. ${ }^{4}$

Outcomes will be examined at both hospital and individual level. The primary outcome measure will be self-harm repetition within 3 months, defined as the proportion of self-harm presentations followed by a subsequent self-harm presentation. Secondary outcomes will include the proportion of biopsychosocial assessments provided at the time of presentation to hospital with selfharm, the proportion of presentations admitted to inpatient ward (medical or psychiatric) and the proportion of presentations receiving a referral following discharge from the emergency department. These outcomes will be examined for a shorter study period, as data are only available for all outcome measures since 2013.

\section{Covariates}

Covariates to be obtained from the Registry will comprise of sociodemographic and clinical variables. Sociodemographic variables will include gender, age, medical card status (whether the individual had access to free medical services, based on income and/or health status) and area of residence. Clinical variables will include method(s) of self-harm, alcohol involvement, drugs taken (if applicable), severity of method, time and day of attendance, self-harm history and clinical management (including receiving a mental health assessment, hospital admission, outpatient referral).

Hospital factors known to be associated with the clinical management and care pathways of self-harm patients will also be used, ${ }^{11}$ including the proportion of self-harm patients who are admitted into the hospital, availability of psychiatric inpatient facilities (onsite or offsite), ${ }^{38}$ hospital location and type of hospital (general or tertiary). ${ }^{39}$ Number of mental health staff employed per 1000 emergency attendances and availability of liaison psychiatry teams will also be included. Data relating to the implementation of the NCP-SH in each hospital will also be gathered, including the date of implementation, number of staff employed and hours of service cover.

\section{Mediators}

The primary implementation outcome will be fidelity to the NCP-SH programme in each hospital, as a mediator between the intervention and the outcomes observed. Aggregate data on patients attending hospital for selfharm are recorded by clinical staff of the NCP-SH and submitted to the programme's Data Manager on a monthly basis. These data include the $6 \mathrm{key}$ indicators of the NCP-SH (outlined in figure 1), which will be averaged to generate a fidelity indicator.

\section{Statistical analyses}

The impact of the implementation of the NCP-SH on intervention outcomes will be formally examined at both hospital and individual level. Initial descriptive analyses will be performed to establish longitudinal trends in the outcomes measures as well as in the overall rate of hospital-presenting self-harm prior to the introduction of the NCP-SH.

At hospital level, changes in outcome measures will be examined using an interrupted time series analysis, ${ }^{40}$ with impacts examined up to 24 months following the introduction of the NCP-SH in each hospital. In addition, survival and logistic analyses will be conducted at the individual patient level, which will detect changes in the likelihood of an outcome measure occurring for an individual, while simultaneously adjusting for individual, hospital and implementation factors. Sensitivity analyses will adjust for periods of implementation, to account for a lead in time for recruitment of staff and protocols being established in hospitals. This time period will be informed by interim site evaluation reports, gathered as part of a review of the operation of the NCP-SH in $2017 .^{20}$

All statistical analyses will be conducted using IBM SPSS Statistics 27 and Stata IC.

\section{Economic evaluation}

A cost-effectiveness analysis will assess the cost per repeat self-harm attendance avoided and the cost per qualityadjusted life-year (QALY) before and after the implementation of the NCP-SH. The cost-effectiveness analysis will be limited to a 12-month period preimplementation and postimplementation in each hospital, taking into account any observed lag in implementation. The perspective of the evaluation will be the health service. Resources will be identified, measured and valued using bottom-up and top-down methods as appropriate. Costs will include provision of mental health assessments, inpatient admission, medical observation unit admission and 
follow-up outpatient department referrals. As QALYs are not collected routinely as part of the Registry or other hospital sources, they will be mapped from existing literature. ${ }^{41}{ }^{42}$ Discounting will be applied to account for the difference in timing between the control period and the intervention period. Cost-effectiveness will be assessed using an Incremental Cost Effectiveness Ratio and a Cost Effectiveness Acceptability Curve.

\section{Stage 2: exploring the implementation of the NCP-SH}

Stage 2 is a qualitative study of the implementation of the NCP-SH, which will include document analysis and semistructured interviews.

\section{Description of the NCP-SH and adopted implementation strategies}

Document analysis will be undertaken using documents that relate to the development and implementation of the NCP-SH, including the programme's standard operating procedures and model of care, along with documents that detail the structure and governance of the NCP-SH (including committee/group terms of reference and meeting minutes). The analysis will also identify the timing and extent of programme implementation across hospital sites, including changes in the model of service and the date in which the programme was implemented in each hospital. Furthermore, it will seek to identify implementation strategies ${ }^{43}$ adopted by individual hospital sites and will be supplemented by interviews with key members of the NCP-SH management team. This will inform the evaluation of the programme in terms of development of the NCP-SH over time as well as the development of an Implementation Research Logic Model. ${ }^{44}$ This model will be used to provisionally map how the relationships between determinants, implementation strategies and mechanisms are potentially related to the implementation and intervention outcomes. The logic model will be revised on completion of the study.

\section{Identification of determinants related to implementation}

Determinants or influences of implementation fidelity will be examined via semi-structured interviews with staff responsible for implementing the NCP-SH in each hospital.

In line with the sequential explanatory design, data from stage 1 will be used to inform the sampling criteria of the semistructured interviews. Hospital sites will be divided into four categories (high intervention/implementation; low intervention/implementation; high intervention/low implementation; low implementation/ high intervention) using average scores from the Incidence Rate Ratios (IRRs) generated from the Poisson regression models (intervention outcomes) and average scores across the 6 indicators of the NCP-SH (implementation fidelity). We will aim to select $50 \%$ of hospital sites, presuming that there will be an even spread across the categories constructed.

Within each hospital, those staff members with responsibility for delivering the NCP-SH (ie, CNSs) will be prioritised for this study. Other key staff members involved at each site include Consultant Psychiatrists, Directors of Nursing and Emergency Medicine Consultants. It is expected that at least 12 CNSs will be recruited, along with 6 participants from each of the other professional groups. Therefore, approximately 30 interviews will be conducted. Potential interviewees will be invited via email or telephone.

\section{Data collection}

The interview guide will be primarily informed by the document analysis and results from stage 1 , as well as by the CFIR. ${ }^{33}$ The CFIR synthesises 39 determinants of implementation organised across 5 domains (Intervention Characteristics; Inner Setting; Outer Setting; Characteristics of Individuals, and Process). The interviews will likely focus on the components of the programme, factors associated with programme implementation in the hospital setting, fidelity to the programme as well as sustainability and resourcing of the programme in the long term. A combination of online and face-to-face interviews will be conducted, to facilitate participants. Participants will be fully informed about the nature and purpose of the interviews and written consent will be obtained. With consent, all interviews will be digitally recorded for transcription in full. Fieldnotes will also be kept by the researcher. Every effort to anonymise participant information and data on individual hospitals collected via these interviews will be made. Pseudonyms will be assigned to all interview data and no identifying information (eg, site name) will be included in the transcribed interviews.

\section{Data analysis}

Data from the semi-structured interviews will be managed using NVivo Software. Data analysis will be conducted using thematic analysis, beginning with the development of a thematic framework. Recordings of the interviews will be listened to while the data are being coded and the thematic framework developed. The themes will be developed iteratively, with merging and division over time. Themes will be compared across groups, to determine whether there are barriers or facilitators unique to specific hospitals or if there are some common across sites.

\section{Data synthesis}

This study will bring together data from two phases of research for the purposes of complementarity, to understand both the impact the programme has had and to identify the factors relating to successful implementation fidelity. ${ }^{31}$ Qualitative findings from the document analysis and interviews with the programme management team will be fed into the interview stage with the clinical staff. The findings from the interviews will be also used to interpret the outcomes observed in the evaluation stage, in particular to explain any observed variation in both the outcome measures and implementation fidelity. Specifically, findings from stages 1 and 2 will be integrated by 
producing a joint display that cross-tabulates the core quantitative outcomes and qualitative themes. ${ }^{45} 46$ This approach will facilitate a complex comparison involving the examination of complementarity, as well as convergence and divergence. ${ }^{46}$

\section{Patient and public involvement}

Patients were not involved directly in the design of this study. It is expected that public and patient involvement will be at the dissemination stage of the research.

\section{Strengths and limitations}

Natural experiment designs have been advocated for use in evaluating the impact of health service initiatives in real-world settings, particularly where randomised control trials are not feasible. ${ }^{32} 47$ Large-scale evaluations such as these are relatively uncommon in the area of suicide research, ${ }^{4-50}$ yet have the potential to examine the impact of complex interventions, particularly those implemented at a population or healthcare level.

This study has several strengths. The adopted design will allow for this study to not only address whether the NCP-SH has been effective in terms of responses to selfharm, but to also identify determinants of implementing multicomponent evidence-based care for self-harm in acute settings. This adds significant value ${ }^{51}$ and will ensure that the findings are relevant to both policy-makers and clinical staff.

There are limitations to the approach taken. Confounding is an inherent problem in natural experiments. We will minimise the issue of confounding through the use of an interrupted time series analysis for the primary outcome, using several years of data to detect any impact due as a result of the programme. For the secondary outcomes, where data are available for a shorter period, a before-and-after study design is the most appropriate method of evaluation, where hospitals act as their own controls. For all analyses, detailed data on implementation of the programme across each site, as well as data on existing services, will be considered. The retrospective nature of the evaluation particularly impacts on the qualitative phase of our study. Potential issues include the turnover of staff since the programme began several years ago, and the accuracy of staffs' ability to recall circumstances of the implementation. To minimise these issues, staff who were in position during the initial stages of implementation will be prioritised. In situations where these staff are no longer in post, it will be required that the staff member participating in the interview should have been in post for at least 12 months.

\section{Ethics and dissemination}

The findings will be important for an international audience, providing an evidence base for routine management of self-harm, as well as highlighting ways to co-ordinate service response across healthcare settings. More generally, few studies have considered the processes underlying delivery of services for self-harm in clinical settings and the relationship between intervention and implementation outcomes has not been well researched. ${ }^{21}$ It is anticipated that this study will lead to a better understanding of how self-harm is managed in acute settings, as well as identifying specific components of clinical care that are related to improved outcomes for patients. Such findings will have the potential to inform further configuration or integration of mental health services in acute settings, in Ireland and internationally. Examining the implementation of these programmes will have learnings for both national and international policy-makers and will contribute to cost-saving initiatives. The findings will lead to a better understanding of how to optimise delivery of services for patients engaging in self-harm and will enable a more comprehensive understanding of how health service change and public health interventions impact on this population.

This study has been approved by the Clinical Research Ethics Committee of the Cork Teaching Hospitals - Reference Numbers ECM 4 (h) 11/5/2021 \& ECM 3 (t) 06/07/2021. Ethical approval for the NSHRI has been granted by the National Research Ethics Committee of the Faculty of Public Health Medicine. The Registry complies with the European Union General Data Protection Regulation and operates a waiver of consent, granted by the Irish Health Research Consent Declaration Committee.

\section{Author affiliations}

${ }^{1}$ School of Public Health, University College Cork, Cork, Ireland

${ }^{2}$ National Suicide Research Foundation, Cork, Ireland

${ }^{3}$ Health Service Executive, Dublin, Ireland

${ }^{4}$ NIHR Biomedical Research Centre, University Hospitals Bristol and Weston NHS

Foundation Trust, Bristol, UK

${ }^{5}$ Population Health Sciences, University of Bristol, Bristol, UK

${ }^{6}$ Department of Economics, Cork University Business School, University College

Cork, Cork, Ireland

${ }^{7}$ Nursing, Midwifery and Allied Health Professions Research Unit, University of Stirling, Stirling, UK

${ }^{8}$ Institute of Health Behaviors and Community Sciences, College of Public Health, National Taiwan University, Taipei City, Taiwan

Twitter Sheena M McHugh @SheMcHugh, David Gunnell @SASHBristol, Ivan J Perry @IvanJPerry, Brendan McElroy @brendanmcelroy3 and Eimear RuaneMcAteer @EimearRMcA

Contributors EG designed the study and wrote the initial draft of the paper. $\mathrm{GC}$ assisted with reviewing the literature, drafting the manuscript and figure development. PC, DG, SMMH, AJ, EA, BM, MM, S-SC and ER-M contributed to the study design and revised the manuscript for publication. All authors read and approved the final manuscript.

Funding This work is funded by the Irish Health Research Board grant number ElA2019-005. DG is supported by the NIHR Biomedical Research Center at University Hospitals Bristol and Weston NHS Foundation Trust.

Disclaimer The funding body have had no role in the study design or writing of the manuscript. The views expressed in this Article are those of the authors and not necessarily those of the Health Research Board.

Competing interests AJ was the Clinical Lead for the National Clinical Programme for the Assessment and Management of Patients presenting to the Emergency Department following Self-Harm (from 1 February 2017 to 31 May 2021).

Patient and public involvement Patients and/or the public were not involved in the design, or conduct, or reporting, or dissemination plans of this research.

Patient consent for publication Not applicable.

Provenance and peer review Not commissioned; externally peer reviewed.

Open access This is an open access article distributed in accordance with the Creative Commons Attribution Non Commercial (CC BY-NC 4.0) license, which permits others to distribute, remix, adapt, build upon this work non-commercially, and license their derivative works on different terms, provided the original work is 
properly cited, appropriate credit is given, any changes made indicated, and the use is non-commercial. See: http://creativecommons.org/licenses/by-nc/4.0/.

\section{ORCID iDs}

Eve Griffin http://orcid.org/0000-0003-0249-6428

Sheena M McHugh http://orcid.org/0000-0002-6595-0491

Anne Jeffers http://orcid.org/0000-0002-4138-208X

David Gunnell http://orcid.org/0000-0002-0829-6470

Ella Arensman http://orcid.org/0000-0003-0376-1203

Ivan J Perry http://orcid.org/0000-0002-4965-9792

Grace Cully http://orcid.org/0000-0002-9236-1545

Brendan McElroy http://orcid.org/0000-0002-0062-330X

Margaret Maxwell http://orcid.org/0000-0003-3318-9500

Shu-Sen Chang http://orcid.org/0000-0001-5012-1132

Eimear Ruane-McAteer http://orcid.org/0000-0003-1669-218X

Paul Corcoran http://orcid.org/0000-0003-1201-7136

\section{REFERENCES}

1 Carroll R, Metcalfe C, Gunnell D. Hospital presenting self-harm and risk of fatal and non-fatal repetition: systematic review and metaanalysis. PLoS One 2014;9:e89944.

2 Bergen $\mathrm{H}$, Hawton K, Waters K, et al. Premature death after selfharm: a multicentre cohort study. The Lancet 2012;380:1568-74.

3 McMahon EM, Keeley H, Cannon M, et al. The iceberg of suicide and self-harm in Irish adolescents: a population-based study. Soc Psychiatry Psychiatr Epidemiol 2014;49:1929-35.

4 Joyce M, Daly C, McTernan N. National Self-Harm Registry Ireland annual report 2019. Cork: National Suicide Research Foundation, 2020.

5 Tsiachristas A, McDaid D, Casey D, et al. General hospital costs in England of medical and psychiatric care for patients who self-harm: a retrospective analysis. Lancet Psychiatry 2017;4:759-67.

6 Steeg S, Emsley R, Carr M, et al. Routine hospital management of self-harm and risk of further self-harm: propensity score analysis using record-based cohort data. Psychol Med 2018;48:315-26.

7 Carroll R, Metcalfe C, Steeg S, et al. Psychosocial assessment of self-harm patients and risk of repeat presentation: an instrumental variable analysis using time of hospital presentation. PLoS One 2016;11:e0149713.

8 Cassidy E, Arensman E, Keeley HS. Saving lives and reducing harmful outcomes: care systems for self-harm and suicidal behaviour. National guidelines for the assessment and management of patients presenting to Irish emergency departments following self-harm. Dublin: Health Service Executive Suicidal Behaviour Working Group, 2012.

9 National Institute for Health and Care Excellence. Self-harm: the short-term physical and psychological management and secondary prevention of self-harm in primary and secondary care. London: National Institute for Health and Care Excellence, 2004.

10 National Institute for Health and Care Excellence. Self-harm quality standard. London: National Institute for Health and Care Excellence, 2013.

11 Griffin E, Gunnell D, Corcoran P. Factors explaining variation in recommended care pathways following hospital-presenting self-harm: a multilevel national registry study. BJPsych Open 2020;6:e145.

12 Cooper J, Steeg S, Gunnell D, et al. Variations in the hospital management of self-harm and patient outcome: a multi-site observational study in England. J Affect Disord 2015;174:101-5.

13 Arensman E, Griffin E, Daly C, et al. Recommended next care following hospital-treated self-harm: patterns and trends over time. PLoS One 2018;13:e0193587.

14 Bridge JA, Olfson M, Caterino JM, et al. Emergency department management of deliberate self-harm: a national survey. JAMA Psychiatry 2019;76:652-4.

15 The Royal College of Emergency Medicine. Mental health in the ED clinical audit 2014-15: national report. The Royal College of Emergency Medicine, 2015.

16 MacDonald S, Sampson C, Turley R, et al. Patients' experiences of emergency hospital care following self-harm: systematic review and thematic synthesis of qualitative research. Qual Health Res 2020;30:471-85.

17 Health Service Executive Mental Health Division. Delivering specialist mental health services 2014-2015. Dublin: Health Service Executive Mental Health Division, 2016.

18 Government of Ireland. A Vision For Change: report of the Expert Group on Mental Health Policy. Dublin, 2006.
19 HSE Clinical Strategy and Programmes Division. National Clinical Programme for the Assessment and Management of Patients Presenting to Emergency Departments Following Self-harm model of care, 2016.

20 Health Service Executive. National clinical programme for the assessment and management of patients presenting to the emergency department following self-harm. review of the operation of the programme. Dublin: Health Service Executive Mental Health Division, 2017.

21 Kapur N. Services for self-harm: progress and promise? Br J Psychiatry 2020;217:663-4.

22 House A, Owens D. General Hospital services in the UK for adults presenting after self-harm: little evidence of progress in the past 25 years. Br J Psychiatry 2020;217:661-2.

23 Griffin E, Murphy C, Perry IJ, et al. Acute hospital reconfiguration and self-harm presentations: a before-and-after study. Ir J Med Sci 2019;188:1-4.

24 Opmeer BC, Hollingworth W, Marques EMR, et al. Extending the liaison psychiatry service in a large hospital in the UK: a before and after evaluation of the economic impact and patient care following ED attendances for self-harm. BMJ Open 2017;7:e016906.

25 Jackson J, Nugawela MD, De Vocht F, et al. Long-Term impact of the expansion of a hospital liaison psychiatry service on patient care and costs following emergency department attendances for self-harm. BJPsych Open 2020;6:e34.

26 Pan Y-J, Chang W-H, Lee M-B, et al. Effectiveness of a nationwide aftercare program for suicide attempters. Psychol Med 2013;43:1447-54.

27 Kawanishi C, Ishii T, Yonemoto N, et al. Protocol for a prospective multicentre registry cohort study on suicide attempters given the assertive case management intervention after admission to an emergency department in Japan: post-ACTION-J study (PACS). BMJ Open 2018;8:e020517.

28 Carroll C, Patterson M, Wood S, et al. A conceptual framework for implementation fidelity. Implement Sci 2007;2:40.

29 Office CS. Census 2016 summary results: Part 1. Dublin: Government of Ireland, 2017.

30 Health Service Executive. National Clinical Programme for the Assessment and Management of Patients who Present to Emergency Department following Self-harm standard operating procedures. Health Service Executive, 2018.

31 Tashakkori A, Teddlie C, eds. SAGE handbook of mixed methods in social \& behavioral research. 2nd ed. California, 2010.

32 Craig P, Cooper C, Gunnell D, et al. Using natural experiments to evaluate population health interventions: new Medical Research Council guidance. J Epidemiol Community Health 2012;66:1182-6.

33 Damschroder LJ, Aron DC, Keith RE, et al. Fostering implementation of health services research findings into practice: a consolidated framework for advancing implementation science. Implement Sci 2009;4:50.

34 Moore GF, Audrey S, Barker M, et al. Process evaluation of complex interventions: Medical Research Council guidance. BMJ 2015;350:h1258.

35 Hoffmann TC, Glasziou PP, Boutron I, et al. Better reporting of interventions: template for intervention description and replication (TIDieR) checklist and guide. BMJ 2014;348:g1687.

36 Perry IJ, Corcoran P, Fitzgerald AP, et al. The incidence and repetition of hospital-treated deliberate self harm: findings from the world's first national registry. PLoS One 2012;7:e31663.

37 Schmidtke A, Bille-Brahe U, DeLeo D, et al. Attempted suicide in Europe: rates, trends and sociodemographic characteristics of suicide attempters during the period 1989-1992. Results of the WHO/ EURO Multicentre Study on Parasuicide. Acta Psychiatr Scand 1996;93:327-38.

38 Health Service Executive. Management data report December 2018. Health Service Executive, 2018.

39 Department of Health. Securing the future of smaller hospitals: a framework for development. Department of Health (DoH), 2013: 2013

40 Wagner AK, Soumerai SB, Zhang F, et al. Segmented regression analysis of interrupted time series studies in medication use research. J Clin Pharm Ther 2002;27:299-309.

41 Owens D, Wright-Hughes A, Graham L, et al. Problem-solving therapy rather than treatment as usual for adults after self-harm: a pragmatic, feasibility, randomised controlled trial (the MIDSHIPS trial). Pilot Feasibility Stud 2020;6:1-14.

42 Quinlivan L, Steeg S, Elvidge J, et al. Risk assessment scales to predict risk of hospital treated repeat self-harm: a cost-effectiveness modelling analysis. J Affect Disord 2019;249:208-15.

43 Powell BJ, Waltz TJ, Chinman MJ, et al. A refined compilation of implementation strategies: results from the Expert Recommendations for Implementing Change (ERIC) project. Implement Sci 2015;10:21. 
44 Smith JD, Li DH, Rafferty MR. The implementation research logic model: a method for planning, executing, reporting, and synthesizing implementation projects. Implement Sci 2020;15:84.

45 Richards DA, Bazeley P, Borglin G, et al. Integrating quantitative and qualitative data and findings when undertaking randomised controlled trials. BMJ Open 2019;9:e032081.

46 Morgan DL. Commentary-After triangulation, what next? J Mix Methods Res 2019;13:6-11.

47 Gunnell D. A population health perspective on suicide research and prevention. Crisis 2015;36:155-60.

48 Cha ES, Chang S-S, Gunnell D, et al. Impact of paraquat regulation on suicide in South Korea. Int J Epidemiol 2016;45:470-9.
49 Godoy Garraza L, Walrath C, Goldston DB, et al. Effect of the Garrett Lee Smith Memorial suicide prevention program on suicide attempts among Youths. JAMA Psychiatry 2015;72:1143-9.

50 Erlangsen A, Lind BD, Stuart EA, et al. Short-term and long-term effects of psychosocial therapy for people after deliberate self-harm: a register-based, nationwide multicentre study using propensity score matching. Lancet Psychiatry 2015;2:49-58.

51 Craig P, Katikireddi SV, Leyland A, et al. Natural experiments: an overview of methods, approaches, and contributions to public health intervention research. Annu Rev Public Health 2017;38:39-56. 